\title{
Theodor Mommsen, die deutschen Professoren und die Revolution von 1848
}

\author{
Stefan Rebenich
}

Ende April 1848 eilte Georg Herwegh an der Spitze von tausend Freiwilligen aus dem französischen Exil in die Heimat zurück - und verlor im südbadischen Dossenbach an der Spitze seiner "Deutschen Demokratischen Legion“ den Kampf gegen die Bundestruppen. Die Niederlage zerstörte alle Hoffnungen auf eine republikanische Revolution. Herweghs Lied „Mein Deutschland, streck die Glieder" war der traurige Abgesang auf das gescheiterte Experiment der nationalen Emanzipation. In seinen Versen strafte er die „fünfhundert Narrenschellen" in Frankfurt ab und verspottete das „Professorenparlament" der Paulskirche: „Die Professoren reißen / nicht Altar noch Thron uns ein, / auch ist der Stein der Weisen / kein deutscher Pflasterstein. "l

In der Tat galten akademisch Gebildete, allen voran Hochschullehrer, im vormärzlichen Bürgertum und noch über die Revolutionsjahre 1848/49 hinaus als „die führenden Autoritäten gesellschaftlicher Selbstdeutung." Die Frankfurter Nationalversammlung bestand zu rund drei Vierteln aus Abgeordneten, die eine Universitätsausbildung erhalten hatten. Auch wenn die neuere Sozialgeschichtsschreibung von der älteren nationalliberalen Revolutionsforschung den Topos des „Professorenparlaments" zu Recht nicht übernommen hat und eher von einem Beamten- oder Juristenparlament spricht, ${ }^{3}$ so steht dennoch außer Frage, daß ,bildungsbürgerliche ${ }^{\mathfrak{A}}$ Abgeordnete den politischen Diskurs nachhaltig beeinflußten und bis weit in das Kaiserreich hinein „die dominante Kultur" prägten. ${ }^{4}$

Einem Vertreter des akademisch gebildeten Bürgertums, der zu den Protagonisten der Revolution von 1848/49 zählte, auch wenn er nicht in der Frankfurter Paulskirche $\mathrm{saB}$, gilt im folgenden unsere Aufmerksamkeit: Theodor Mommsen, der als Journalist und als Professor für römisches Recht in die Zeitlăufte eingriff. An seiner Vita, „gleichsam im Medium der Wahrnehmung und Beurteilung" eines Beteiligten, soll ein Zugang zur Geschichte des Liberalismus wăhrend der Revolution von $1848 / 49$ gefunden werden. ${ }^{6}$ Dabei interessieren insbesondere die revolutionären Vorgänge in Schleswig-Holstein und 
in Sachsen, in die Mommsen unmittelbar involviert war. Seine theoretischen Anschauungen und praktischen Forderungen, seine Zielsetzungen und Aktionen, seine Radikalisierung und seine Resignation, seine Entwicklungen und Regressionen, seine Bewältigung des Traumas der gescheiterten Revolution und seine politischen Folgenungen werden auf den folgenden Seiten darzustellen sein. Darüber hinaus ist seine revolutionăre und postrevolutionăre Biographie mit der anderer Universitätsprofessoren zu vergleichen. Ziel dieser Ausführungen ist es, an Mommsens Beispiel den Typus eines liberalen Hochschullehrers scharf zu konturieren.

Ich beginne mit Mommsens Einstieg in die revolutionäre Bewegung nach seiner Rückkehr aus Italien (I), untersuche die Ziele und Inhalte, die er als Journalist in Rendsburg verfolgte (II), betrachte sein politisches Engagement in der sächsischen Universitătsstadt Leipzig (III), zeichne die Abstrafiung des Liberalen durch Straf- und Disziplinarrecht nach (IV) und frage schlieBlich nach den langfristigen Wirkungen der Revolutionserfahrung in Mommsens Biographie (V).

\section{Der Italienheimkehrer}

Im Frühjahr 1843 bestand Mommsen, der 25jährige Pfarrerssohn aus Garding, nach zehnsemestrigem Studium sein juristisches Examen mit Auszeichnung. Im November desselben Jahres wurde er über ein römischrechtliches Thema summa cum laude promoviert. Doch die hochgesteckten beruflichen Ziele ließen sich nicht verwirklichen, wie sich schnell zeigte. So war an eine akademische Karriere vorerst nicht zu denken. Mommsen muBte sich zunächst als Aushilfslehrer in den Mädchenpensionaten seiner Tanten in Altona über Wasser halten. Ein Zubrot verdiente er sich als Journalist. Er schrieb für verschiedene Blătter politische und literarische Beiträge und berichtete gelegentlich von Hamburger Theateraufführungen. ${ }^{8}$

Im April 1844 erhielt Mommsen ein Reisestipendium des dänischen Königs und brach nach Italien auf. Dort studierte er Inschriften und legte die Grundlagen für eine umfassende lateinische Inschriftensammlung, die nach langem Ringen und vielen Auseinandersetzungen 1854 schließlich von der Berliner Akademie übernommen wurde. Das ursprüngliche romanistische Ziel seiner Stipendienreise, alle inschriftlich erhaltenen Gesetzesurkunden zu sammeln, war dem großen Plan gewichen, ein für die Altertumsforschung grundlegendes Corpus Inscriptionum Latinarum herauszugeben. ${ }^{9}$ Mommsen selbst 
brachte es bei seiner Ansprache zu seinem 60. Geburtstag auf den Punkt: „Der Jurist ging nach Italien - der Historiker kam zurück. " ${ }^{\text {10 }}$

Doch als er im Sommer 1847 in die Heimat zurückkehrte, erwartete ihn dort die Arbeitslosigkeit. ${ }^{11}$ Es nützte Mommsen herzlich wenig, daB er sich inzwischen unter Altertumswissenschaftlern einen großen Ruf erworben hatte. Jetzt stand er mittellos da. Der junge Gelehrte wechselte wieder an die Schule. Eben noch hatte er in Rom vor internationalem Publikum Inschriften interpretiert, nun unterrichtete er im Altonaer Institut der Tanten Mädchen im heiratsfähigen Alter. Die Zukunft war ungewiB. Doch schon bald ließen ihn die politischen Ereignisse die berufliche Tristesse vergessen.

In den Herzogtümern Schleswig und Holstein gärte es. Aus dem regionalen Gegensatz zwischen den Herzogtümern und dem dänischen Staat war eine nationale Sache geworden, die ganz Deutschland bewegte. Erinnern wir uns: Seit den 1830er Jahren wurde immer energischer eine gemeinsame Verfassung für Schleswig und Holstein gefordert und für eine Lockerung der Bindung an Dänemark gestritten. Die Regierung in Kopenhagen ließ sich indes nur zur Einrichtung von getrennten Provinzialständen bewegen, betrieb ansonsten in Schleswig eine geschickte Danisierungspolitik und unterstützte die radikalen Vertneter der dänischen Minorität, die offen für den Anschluß des Herzogtums an Dänemark agitierten. Dagegen formierte sich Widerstand. Schleswig und Holstein sollten als deutsche Länder ungeteilt bleiben. Die alte Vertragsformel „up ewig ungedeelt“ wurde zum politischen Schlachtruf, und aus vielen Kehlen ertönte das nationale Kampflied "Schleswig-Holstein meenumschlungen ${ }^{\star 12}$

Die Spannungen verschärften sich 1846, als der dänische König Christian VIII. in den Herzogtümern das Erbfolgerecht der dänischen Gesamtmonarchie einführte. Selbst unpolitische Köpfe wie Theodor Storm ließen sich daraufhin zu Protestaktionen hinreißen. ${ }^{13}$ Die Nachrichten von der Pariser Februarrevolution heizten Anfang 1848 die Krisenstimmung zusätzlich an. In Schleswig und Holstein wurden wie überall in Deutschland ,Mărzforderungen' erhoben: Volksbewaffnung, Schwurgerichte, Presse- und Versammlungsfreiheit. Am 18. März verlangten die Deputierten in Rendsburg die Zusammenlegung der holsteinischen und schleswigschen Ständeversammlungen, eine gemeinsame Verfassung und die Aufnahme Schleswigs in den Deutschen Bund. Schon bildeten sich Freiwilligenverbände, die mit der Waffe in der Hand für ihre Ziele zu streiten bereit waren. Auch Mommsen, der sich eben noch in Hamburg „bei einem elenden Krawall" das Ohr hatte „abhauen lassen " ${ }^{44}$ meldete sich in Rendsburg für ein Freikorps, wurde aber, weil dienstuntauglich, abgewiesen. Die Berufung eines neuen, vorwiegend aus Nationalliberalen gebildeten Ministeriums im Februar 1848 durch den neuen Monarchen, Friedrich VII., 
verschärfte die Gegensătze, denn in Kopenhagen beharrte man auf der Angliederung Schleswigs an Danemark. Daraufhin wurde in Kiel eine provisorische Regienung gebildet, deren erklărtes Ziel es war, für die nationale Einheit und Freiheit Deutschlands und die Aufnahme Schleswigs in den Deutschen Bund zu streiten. Am 23. März erklärten die Herzogtümer Schleswig und Holstein ihren Abfall von Dänemark. Die Festung Rendsburg wurde besetzt und zum Regierungssitz ausgerufen. Der Aufstand war ausgebrochen.

Mitglied der in Frankfurt und Berlin anerkannten provisorischen Regierung war Theodor Olshausen, der im „Kieler Korrespondenzblatt“ zeitweilig für die Abtrennung Schleswigs von Holstein eingetreten war, sich nun aber zu einem eifrigen Verfechter der Unabhängigkeit und Einheit der beiden Herzogtümer gewandelt hatte. Der populäre liberale Politiker mit journalistischer Erfahrung leitete mit der gerade gegründeten „Schleswig-Holsteinischen Zeitung ein Presseorgan, das die offizielle Politik der neuen Regierung in den Herzogtümern verbreiten sollte. Da er dem alten Schriftleiter wenig zutraute, schaute er sich nach einem geeigneteren Redakteur um und gewann Mommsen, den Olshausen aus gemeinsamen Kieler Zeiten kannte und schätzte. ${ }^{15}$.

Am 18. April bezog Mommsen die Redaktionsstube. Schon am 23. April erlebte er bei Schleswig als, journalistischer Schlachtenbummler" den Sieg der preuBischen Truppen, die die Aufstăndischen gegen Dänemark unterstützten. ${ }^{16}$ Am nachsten Tag handelte er über die Wahlen zum Frankfurter Parlament, ${ }^{17}$ um am 25. April einer Versammlung des Wahlkommitees in Neumünster beizuwohnen. Energisch unterstützte er die Kandidatur von Johann Gustav Droysen und Georg Waitz, während er den Staatsrechtler Lorenz Stein aus Kiel niederschrieb. ${ }^{18}$

\section{Der Journalist}

Mommsen setzte sich als Journalist energisch für die politische Profilierung der „Schleswig-Holsteinischen Zeitung“ ein. Er hatte zwar das Journalistenhandwerk nicht gelernt, aber er formulierte glänzend. Lokale, regionale und nationale Ereignisse wurden in seinen Artikeln besprochen, die parlamentarischen Debatten in den Landesvertretungen und in der Frankfurter Paulskirche dokumentiert, Aufrufe, Petitionen und Denkschriften abgednckt und die Leser zur Diskussion aufgefordert. Der Journalist Mommsen war bei der ,Kommunikationsrevolution ${ }^{4}$ von 1848 dabei: ${ }^{19}$ Nicht durch parlamentarische Reden oder politische Druckschriften trug er zur Politisienung der Öffentlichkeit bei, sondern durch brillant formulierte Leitartikel. Mommsen stand - wie die Parla- 
mentarier Friedrich Christoph Dahlmann, Karl Theodor Welcker, Johann Gustav Droysen und Georg Waitz - mitten im politischen Geschehen. Aber es gelang ihm nicht, der Zeitung überregionale Bedeutung zu verschaffen. Die Zahl der Abonnenten kam über 2.000 nicht hinaus. Der Leserstamm entsprach damit dem anderer und weitaus farbloserer Lokalblätter.

Mommsen vertrat in der "Schleswig-Holsteinischen Zeitung“ die liberale Politik der Mitte. Einen forschen Patriotismus, der sich gegen Dänemark richtete, verband er mit dem glühenden Bekenntnis zur deutschen Einheit. „Ungeteilt" hieß die Devise. Die Entscheidung über das Schicksal seiner Heimat mußte seiner Ansicht nach in der Frankfurter Paulskirche fallen. Das romantische Ideal der deutschen Kulturnation, das schon Barthold Georg Niebuhr sich zu eigen gemacht hatte, ${ }^{20}$ verschmolz er mit der liberalen Idee einer „Staatsnation". Mommsen war von der Lebensunfähigkeit der deutschen Kleinstaaten überzeugt und setzte alle seine Hoffnungen auf Preußen. „Wir anderen Deutschen brauchen Preußen notwendiger, als Preußen uns", floß es ihm damals in die Feder. ${ }^{21}$ Preußens Mission war die Errichtung des deutschen Nationalstaates. Wie Johann Gustav Droysen forderte auch Mommsen 1848, daß Preußen sein Staatsinteresse mit dem Interesse der deutschen Nation identifizieren und letztlich in Deutschland aufgehen solle. ${ }^{22}$ Konsequent wird Mommsen nach der dänischen Niederlage bei den Düppeler Schanzen im Jahre 1864 der preuBischen Annexion Schleswig-Holsteins seinen Beifall bekunden. ${ }^{23}$ „Militanter Nationalismus und konstitutionelle Rechtsordnung ${ }^{\text {c24 }}$ waren auch für Mommsen keine Gegensätze. Im Unterschied zu vielen süddeutschen Liberalen ließ er aber nie einen Zweifel daran, daB er einen kleindeutsch-preuBischen Bund, der Österreich ausschloB, favorisierte.

Mommsen kommentierte in der „Schleswig-Holsteinischen Zeitung " nicht nur die Entwicklung in den beiden Herzogtümern, sondern auch die großen Fragen, die das Frankfurter Vorparlament und die verfassungsgebende Nationalversammlung bewegten. ${ }^{25}$ In seinen Artikeln zur Zentralisierung und zum Erbkaisertum, zur Repräsentation des Volkes und zu Wahlrechtsfragen, zur Gewaltenteilung und zum Verhältnis von Regierung und Parlament setzte er sich mit den unterschiedlichen politischen Strömungen auseinander, suchte nach liberalen Antworten auf die drăngenden Fragen der Zeit und präzisierte nicht immer frei von Widersprüchen - seine politische Aussage. Dabei war er ein unabhängiger Kopf, der sich in keine Parteischablone pressen lieB. Er sympathisierte oft mit Positionen der linken Mitte. „Keine Isolirung! Keine Reaction! Keine Anarchie!" lautete sein Wahlspruch. ${ }^{26}$

Entschieden wandte er sich gegen separatistische Strömungen und versagte - zunächst zumindest - den Nordschleswigern, unter denen sich zahlreiche 
Dänen befanden, das Recht auf nationale Selbstbestimmung. Das ganze Land sollte deutsch sein. Gleichzeitig agitierte er gegen fürstliche Reaktion und sozialistischen Umsturz. Reformen wurden begrüBt, aber Legalität und Rechtskontinuität eingefordert. Nicht Freiheit vom Staat, sondern im Staat lautete seine Devise. Die konstitutionelle Monarchie war Mommsen die sicherste Gewảhr gegen Anarchie, die die Liberalen seit den Exzessen der Französischen Revolution fürchteten wie der Teufel das Weihwasser. Monarchie und Parlamentarismus galten ihm als vereinbar. Die von den Demokraten geforderte Republik mit einem souverănen Nationalparlament schloB er zwar für die Zukunft nicht prinzipiell aus, aber im Moment schien ihm die Einigung Deutschlands unter einem preuBischen Erbkaiser taktisch geboten. Im Zentrum seiner liberalen Repräsentationstheorie standen die gewählten Abgeordnetenkammern, durch die das Bürgertum an den Staatsgeschäften, vor allem der Steuerbewilligung und der Gesetzgebung, mitwirken und die Regierungen kontrollieren sollte. Auf diese Weise sah er das Prinzip der Volkssouverănität garantiert: Die Nation muß und soll sich selbst regieren. Die liberale Rechtsstaatsidee als Grundlage einer bürgerlichen Gesellschaft wurde Mommsen in dieser Zeit so wichtig, daß er einzelne Elemente selbst in historischen Formationen wie dem Staatsrecht des republikanischen Rom suchte. ${ }^{27}$

Mommsens Leitbild war eine klassenlose, ,mittelständische' Gesellschaft gleichberechtigter Staatsbürger, in der soziale Gegensătze ausgeglichen wurden. Die ständisch-feudale Privilegiengesellschaft war seine Sache nicht, genausowenig die kapitalistische Klassengesellschaft englischer Provenienz, die etwa Gervinus propagierte. ${ }^{28}$ So widmete Mommsen der "Verbesserung der Lage der arbeitenden Klassen" einen Artikel. ${ }^{29}$ Sicherheit und Stabilität der offentlichen Verhältnisse waren seine erste Sorge, aber er setzte sich auch für den vorsichtigen Wandel des historisch Gewordenen ein. In einer auf Vernunft gegründeten Gemeinschaft freier Individuen würden materielle Ungleichheiten und soziale Diskriminierung zwar nicht vermieden, aber wenigstens eingeschränkt, und den Arbeitern würde die Möglichkeit eröffnet, sich durch den Erwerb von Bildung und Besitz aus ihrer Notlage zu emanzipieren und an der bürgerlichen Freiheit zu partizipieren. Mommsen war mithin empfänglich für die Konzeption einer sozial und wirtschaftich modernisierten Gesellschaft ohne ständisch-korporative Bindungen, die Hegel theoretisch fundiert hatte. Aber die Reform der bürgerlichen Gesellschaft sollte die Herrschaft des Bürgertums nicht grundsätzlich in Frage stellen.

Anfang Juni 1848 freute sich Mommsen, daB es Dahlmann, Droysen, Waitz und anderen nationalliberalen Vertretern der Herzogtümer im Frankfurter Parlament gelungen war, die schleswigsche Sache zu einer deutschen Sache 
zu machen. ${ }^{30}$ Doch schon wenig später stand Mommsen in Rendsburg im Abseits, da er die Vereinigung der holsteinischen und schleswigschen Ständeversammlungen und einige Abgeordnete scharf kritisiert hatte. Er sah in ihnen „nicht die Repräsentation des Volkes“, sondern ein Hemmnis des „freisinnigen Fortschritts" und forderte die Einführung der allgemeinen Wehrpflicht und eines liberalen Wahlgesetzes. ${ }^{31}$ Die Zahl seiner Gegner wuchs in der ohnehin mehrheitlich konservativen Regierung. Als ihm ein Maulkorb verpaßt werden sollte, trat er in den ersten Julitagen von seiner Stelle als Schriftleiter zurück. ${ }^{32}$ Er wählte die Arbeitslosigkeit, um der Zensur zu entgehen.

Im August 1848 ließ er sich dazu bewegen, nochmals für die Zeitung zu schreiben. Jetzt war das Blatt privatisiert, und Mommsen machte sich Hoffnungen, daß nicht mehr gouvernementale, sondern unabhängige Berichterstattung gefragt sei. Aber die Zeitlăufte waren widrig. Preußen fürchtete die Internationalisierung des deutsch-dänischen Konfliktes und schloB im schwedischen Malmő mit Dänemark einen Waffenstillstand. Die Entscheidung wurde in Rendsburg wie in Frankfurt kontrovers diskutiert. Während die Demokraten die Vereinbarung von Malmö kategorisch ablehnten, rang sich Mommsen schließlich - wie viele seiner liberalen Freunde - zur Zustimmung durch. Nun billigte er auch die Teilung des Herzogtums Schleswig, die er im Juni noch zurückgewiesen hatte. Nur so glaubte er, den großeren Teil des Herzogtums für den Deutschen Bund retten zu können. ${ }^{33}$ Sein Glaube an den Primat der Nationalversammlung hatte jedoch in diesen Auseinandersetzungen deutlich an Kraft verloren. Bald sprach er abfällig von der Frankfurter „Parlamentsidyl$1 e^{\text {" }}$, die ihn an den Vogelstaat des Aristophanes erinnerte. ${ }^{34}$ Die journalistische Arbeit war ihm vorerst jedoch verleidet. Anfang Oktober schrieb er seinen letzten Artikel.

\section{Der Universitätsprofessor}

Nachdem Mommsen Ende Juni 1848 die Schriftleitung der „SchleswigHolsteinischen Zeitung " niedergelegt hatte, versuchte er zunächst, in Frankfurt als Journalist unterzukommen. Einige Tage bezog er bei Droysen in Frankfurt Quartier, um sich in den politischen Kreisen umzusehen. Er hoffte auf eine

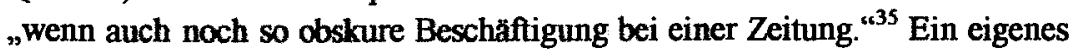
Blatt zu grilnden, wie Olshausen ihm geraten hatte, traute er sich nicht zu. Hilfe kam indes von anderer Seite. Der geschickten Verhandlung seines alten Lehrers und treuen Freundes Otto Jahn, der seit 1847 eine Professur für Archăologie und Philologie an der Universităt Leipzig innehatte, war es zu ver- 
danken, daß der słchsische Kultusminister Ludwig von der Pfordten, der selbst eine Zeitlang Ordinarius für Römisches Recht in Würzburg gewesen war, den stellungslosen Wissenschaftler als außerordentlichen Professor der Rechte zum Wintersemester 1848/49 nach Leipzig berief. Mommsen war heilfroh, "dem Journalisieren " entkommen zu sein, und akzeptierte ohne Zögern das niedrige Einstiegsgehalt von 400 Talern. $^{36}$

Ende September traf er in Leipzig ein. ${ }^{37}$ Die Universität war mit knapp 1.000 Studenten eine der größten in Deutschland. Die Juristenfakultät florierte. Seine Antrittsrede hielt Mommsen an einem der letzten Oktobertage über die Aufgaben der historischen Rechtswissenschaft. Er war zurück im akademischen Milieu. Doch Leipzig bedeutete zugleich die Fortsetzung seines politischen Engagements. Schon als ihm Jahn die frohe Kunde vom Ruf nach Leipzig überbrachte, gab er dem Freund gleich noch ein paar gutgemeinte Ratschläge mit auf den Weg: „Freilich darfst Du nicht erst mit Blum Freundschaft schließen und muBt Deine Sympathien für die Linke bis zum linken Zentrum ermäBigen, Dich an den gutmütigen Fortschritt des Deutschen Vereins gewöhnen, und überhaupt für den deutschen Salat mehr Öl als Essig bringen, wenn Du Dich hier behaglich fühlen willst. ${ }^{\text {c38 }}$ Mommsen lernte in Leipzig nicht nur Robert Blum kennen, den überzeugten Republikaner und entschiedenen Führer der Linken im Frankfurter Parlament. In der Messestadt gab es, als Mommsen im Herbst 1848 eintraf, ein differenziertes politisches Vereinswesen, das die Strömungen der Zeit spiegelte. Wie überall in Deutschland so organisierten die Vereine auch in der sächsischen Handelsmetropole die politische ,Basis‘. Auf der linken Seite stand in Leipzig der „Vaterlandsverein“" der Demokraten, der sich schon früh zur auBerparlamentarischen Opposition bekannte und für die uneingeschränkte Umsetzung der Mărzforderungen, weitgehende Grundrechte und eine republikanische Verfassung eintrat. Im „Vaterlandsverein ${ }^{\star}$ versammelten sich nicht nur Professoren und Freiberufler, sondern auch Handwerksgesellen und Arbeiter.

Der „Deutsche Verein" hingegen war die Organisation der Liberalen oder der „Konstitutionellen“, wie man damals sagte. Ihm trat Mommsen unmittelbar nach seiner Ankunft bei. Dort engagierten sich seine Leipziger Freunde, die Mommsen durch Otto Jahns Vermittlung kennengelernt hatte und die bïrgerliche Herkunft und liberale Gesinnung miteinander verband. So lernte Mommsen den fast zehn Jahre älteren Philologen Moriz Haupt kennen, der Schüler und Schwiegersohn des berïhmten Gottfried Hermann war und seit 1843 den neu geschaffenen Lehrstuhl für deutsche Sprache und Literatur in Leipzig innehatte. Auch Julius Ludwig Klee gehörte zu den neuen Freunden. Er ging 1848 als Rektor der angesehenen Kreuzschule nach Dresden, ließ aber seine 
Verbindungen zum Leipziger Kreis nicht abreißen. Die drei Professoren Haupt, Jahn und Mommsen und der Gymnasiallehrer Klee trafen sich regelmäßig mit den Verlegern Karl Reimer und Salomon Hirzel, den Besitzern der Weidmannschen Buchhandlung, ${ }^{39}$ sowie Hermann Härtel und Georg Wigand, in dessen Haus Mommsen und Jahn wohnten. Ein solcher Kontakt verstand sich in Leipzig, der Stadt des Buchhandels, fast von selbst. ${ }^{40}$

Den Verlegern Härtel und Wigand, Reimer und Hirzel kam bei der publizistischen Offensive des sächsischen Liberalismus eine wichtige Rolle $\mathrm{zu}$, da sie über weitreichende Verbindungen verfügten. So war Karl Reimer mit Friedrich Dahlmann befreundet. Nachdem der liberale Historiker 1837 als einer der „Sieben" aus Göttingen vertrieben worden war, fand er monatelang Zuflucht im Haus des Verlegers, der zusammen mit Salomon Hirzel zu den zehn Mănnern gehörte, die am 9. Dezember 1837 in Leipzig den „Göttinger Verein “ ins Leben riefen, um den sieben relegierten Göttinger Professoren materielle Unterstützung zukommen zu lassen.

Im „Deutschen Verein“ fanden sich als Wortfürer des liberalen Bürgertums zahlreiche Professoren der Leipziger Universität. ${ }^{41}$ Gemeinsam mit Haupt, Jahn, Klee und Mommsen vertraten der Theologe Gustav Fricke, der Jurist Hermann Schletter sowie die Historiker Ludwig Flathe und Heinrich Wuttke das Programm des Vereins, dessen Organ die seit dem 1. August 1848 erscheinenden „Deutschen Blătter“ waren. Haupt und Klee waren zwei der drei Herausgeber, Mommsen schrieb regelmäBig Beiträge. Als Mommsen nach Leipzig kam, wurde in dem mitgliederstarken Verein und seiner Zeitung heftig über den Aufbau und die Verfassung eines vereinten Deutschlands gestritten. Einigkeit herrschte darüber, daß die Einzelstaaten konstitutionelle Monarchien sein sollten, die „auf breitester demokratischer Grundlage“ ruhen müBten. ${ }^{42}$ Über die Führung und Struktur des als Bundesstaat geeinten Deutschlands wurde ebenso heftig debattiert wie über die Frage, ob die parlamentarische Repräsentation auf einem Ein- oder Zweikammersystem beruhen sollte. Schon Anfang Juli 1848 hatten sich die Vertreter einer stärker monarchistischen Richtung abgespalten und den „Deutschen Konstitutionellen Verein" ins Leben gerufen. Haupt und Fricke, die bald von Mommsen unterstützt wurden, gingen auf Distanz sowohl zu dieser schismatischen Gruppierung wie zum "Vaterlandsverein“, beriefen sich trotzig auf die fortschrittlichen Grundsătze der Volkssouverănităt und schwenkten im übrigen auf die erbkaiserliche Linie ein. Eine republikanische Präsidentschaft oder ein Wahlkaisertum wollten sie nicht. Damit wurde zugleich den großdeutschen Hoffnungen eine Absage erteilt und die preubische Option gewthlt. Mommsen blieb seiner Linie treu. 
Die demokratische Linke hielt sich mit Angriffen auf die liberale Mitte nicht zurück. Der Waffenstillstand von Malmö (26. August 1848), der zunächst vom Nationalparlament in der Paulskirche verworfen, dann aber gebilligt worden war, hatte nicht nur in Frankfurt für Unruhen gesorgt. Auch in Sachsen gerieten das rechte und linke Zentrum unter BeschuB. Die Leipziger Gelehrten im „Deutschen Verein“" wurden als „vertrocknete Philologen" lächerlich gemacht, die jahrelang an der „Entzifferung eines Tintenklecks ${ }^{\iota}$ arbeiteten, statt ihren Studenten die „großen republikanischen Tugenden“ der Alten beizubringen. ${ }^{43}$ Die Lage war angespannt. Da traf die Nachricht vom Tode Robert Blums ein. Der Politiker war im Oktober nach Wien gereist, um dort die Aufständischen in ihrem Kampf gegen die österreichische Regierung zu unterstützen. Nach der Einnahme Wiens durch Windischgrätz wurde er am 9. November 1848 standrechtlich erschossen. Blum wurde zum Märtyrer der Demokraten. Sie gewannen die Wahlen für die sächsische Volksvertretung im Dezember 1848 haushoch. Mommsen zeigte sich schockiert. Haupt, der für die Liberalen kandidiert hatte, fiel durch. In der Universität war den Mitgliedern des „Deutschen Vereins" der Spott der demokratischen Studenten sicher.

Der „Deutsche Verein“ ging zunächst publizistisch in die Offensive. Anfang 1849 verfaßte Mommsen einen Kommentar zu den in Frankfurt ausgearbeiteten und verabschiedeten Grundrechten des deutschen Volkes, ${ }^{44}$ die von der äußersten Linken als „Trümmer“ der Revolution abgelehnt wurden. Mommsen hingegen erblickte in ihnen die Garantie bürgerlicher Freiheit und die notwendige Basis für die nationale Einigung. Seine Schrift wurde zum Bestseller. Innerhalb kürzester Zeit gingen 10.000 Exemplare über den Ladentisch. Als der von den Demokraten majorisierte sächsische Landtag sich in einer kontroversen Debatte am 20. Januar 1849 gegen das Erbkaisertum aussprach und damit aus der Perspektive des „Deutschen Vereins“ partikularistischen Tendenzen Vorschub leistete, richtete der „Deutsche Verein“ drei Tage später eine Adresse an den Minister von der Pfordten, in der mit der Volksvertretung abgerechnet wurde: „Ist auch der souveräne Unverstand für den Augenblick zur Herrschaft gelangt, so sind doch seine Tage gezählt; die betörte Mehrheit im Volke wird die Augen offnen und endlich begreifen, daB nur in und mit Deutschland für unser sächsisches Land eine bessere Zukunft gedeihen kann. “45 Das als "Unverstandsadresse“ berühmt gewordene Pamphlet hatte wiederum Mommsen geschrieben. Die „radikale Partei“ erboste sich daraufhin „gelegentlich in ihren Organen über den blutjungen meerumschlungenen Professor", wie Mommsen an seinen Bruder Tycho schrieb. ${ }^{46}$ In der Hoffnung, die Einheit Deutschlands zu retten, plädierte er, um das zeitweise partikularistische Parla- 
ment zu schwăchen, für ein Bündnis mit der grundsätzlich partikularistischen Regierung. Diese Taktik konnte nicht aufgehen.

Die Regierung, die in Übereinstimmung mit dem König handelte, dachte nämlich keineswegs daran, die Grundrechte in Sachsen zu garantieren. Über die săchsischen Vorbehalte gegen Preußens Führungsrolle in der Einheitsbewegung zeterte Mommsen privat und öffentlich, in deutschen und lateinischen Invektiven. Eine Annäherung an die Demokraten schien jetzt angeraten, um die Regierung zur Annahme der Reichsverfassung zu zwingen, über die gerade in Frankfurt beraten wurde. Dafür nahmen Haupt, Jahn und Mommsen den Bruch mit den liberalen sächsischen Abgeordneten in Kauf, die den drei Professoren vorwarfen, ins radikale Lager übergelaufen zu sein.

Die nationale Verfassungsfrage rückte in den Brennpunkt des politischen Geschehens. Haupt, Jahn und Mommsen drängten in Wort und Schrift auf eine Beschleunigung der Beratungen um die Reichsverfassung. Am 27. März 1849 schienen sie ihr Ziel erreicht zu haben: Im Frankfurter Parlament wurde der Erbkaiserplan mit 267 gegen 263 Stimmen angenommen. Die großdeutsche Lösung war endgültig abgelehnt. Auch Mommsen und seine Freunde blickten auf den preußischen König Friedrich Wilhelm IV., der zum Kaiser gewăhlt wurde. Doch der lehnte die „mit dem Ludergeruch der Revolution behaftete“ Würde ab und löste am 27. April die preußische zweite Kammer auf. Am selben Tag forderte der ,Deutsche Verein“" in einem Antrag an Rat und Stadtverordnete von Leipzig, das Palladium der deutschen Einheit mit Mut und Entschlossenheit zu verteidigen. Noch klammerte man sich an das Ziel, daß die Verfassung in Sachsen eingeführt werden könnte, aber die Stimmung war denkbar schlecht. „Ich bin in dem Grade verstimmt, erschreckt, erbittert durch die neuesten Nachrichten aus Berlin", schrieb Mommsen am 29. April 1849 an Tycho, „daß ich mich zum Schreiben zwingen muß; Du weißt, innerliches Leiden macht mich stumm. Wenn unser armes Vaterland doch untergehen soll, so hätten wir doch wohl einen Todeskampf und einen Alexander verdient. "47 Am 30. April löste der sächsische Minister Beust den Landtag auf, Am 3. Mai brannten in Dresden die Barrikaden.

In Leipzig wurde hektisch beraten. ${ }^{48}$ Die verschiedenen Vereine der Stadt bereiteten ein Aktionsbündnis vor. Mommsen, Jahn und Haupt vertraten den "Deutschen Verein" in dem neu geschaffenen vereinigten Ausschuß, an dem sich auch weiter links stehende Gruppen beteiligten. Eine Deputation wurde an den Stadtrat gesandt. Sie forderte, die Kommunalgarde mit bewaffneten Bürgern zu verstärken und den Dresdner Aufständischen zu Hilfe zu eilen. Als man damit nicht durchdrang, zogen Mommsen, Jahn und Haupt mit dem Ruf „Bürger heraus!“ durch die Straßen und forderten Passanten auf, sich zu einer 
Volksversammlung einzufinden. ${ }^{49}$ Gleichzeitig wurden Gewehre und Munition herbeigeschafft. Pulverdampf lag in der Luft.

Das Bündnis wăhrte indes nicht lange. Schon am Nachmittag des 4. Mai kündigten die Professoren des „Deutschen Vereins“ die weitere Zusammenarbeit auf. Ihre Solidarităt galt nun den Behörden und der Kommunalgarde. Diese Volte leitete das Ende der Leipziger Maierhebung ein, bevor sie richtig begonnen hatte. Nur eine kleine Schar von Unentwegten versuchte, den Aufstand fortzufuihren, wurde jedoch in der Nacht vom 6. zum 7. Mai zusammengehauen. Wie erklärt sich der auffällige Sinneswandel von Mommsen und seinen Gefahrten? Haupt hat, als er sich später vor Gericht verantworten mußte, sein Verhalten als einen "Akt leidenschaftlicher Aufwallung "bezeichnet. ${ }^{50}$ In der Tat scheint die Nachricht von dem bevorstehenden Einsatz preußischer Truppen in Dresden auch die Mitglieder des „Deutschen Vereins“ in Rage versetzt zu haben. Als es dann ans Kămpfen ging, versagte ihnen der Mut. Sie bezahlten nur noch einen Teil der Waffen und besannen sich dann auf den Grundsatz, daß Gewalt als Mittel der Politik nichts tauge. Hinzu kam, daß die provisorische Regierung, die die Aufständischen in Leipzig nach der Flucht von König und Kabinett noch am 4. Mai gebildet hatten, eine radikale Richtung vertrat, die Mommsen, Jahn und Haupt nicht zusagte. Sie fürchteten wie Liberale andernorts, daß die Linke die Verfassung nur als ,Aushängeschild gebrauchen würde, um eine demokratische und soziale Republik zu schaffen, und antworteten mit einer Loyalitätserklärung für die sächsische Landesverfassung - und damit für die Monarchie.

\section{Der Verurteilte}

In Dresden kämpften der Architekt Gottfried Semper, der Komponist Richard Wagner, der Gymnasiallehrer Hermann Köchly, der sächsische Radikale Samuel Erdmann Tzschirner und der russische Anarchist Michail Bakunin mit einigen Hundert Handwerkern, Arbeitern und Studenten auf verlorenem Posten. Am 5. Mai trafen die preußischen Einheiten ein, die in viertägigen blutigen Straßen- und Hăuserkämpfen die Insurrektion liquidierten. Über 250 Tote waren zu beklagen, und 727 Revolutionäre wurden in den nachfolgenden Prozessen zu Zuchthausstrafen verurteilt. ${ }^{\text {s1 }}$

Doch auch Haupt, Jahn und Mommsen entkamen der Reaktion nicht, weder politisch noch strafrechtlich. Nachdem die Maiaufstände blutig niederkartätscht worden waren, hieB es, in der veränderten politischen Landschaft seinen Standort zu bestimmen. Der „Deutsche Verein“ war nach den Ereignissen 
von Anfang Mai 1849 Angriffen von rechts und links ausgesetzt. Mommsen, dem erbkaiserlich-kleindeutsch orientierten Liberalen, fehlte nach der Verweigerung des preußischen Königs eine realistische Perspektive. Doch das Ringen um die Verfassung und die Gestalt Deutschlands ging auch nach der gescheiterten Revolution weiter. Mommsen zog sich im Gegensatz zu anderen resignierten liberalen Hochschullehrem nicht aus der Politik in das Refugium der Wissenschaft zuräck. Den „Kampf um Sein oder Nichtsein der echten deutschen Nation ${ }^{452}$ führte er weiter.

Die Pläne, die Joseph Maria von Radowitz, ein Vertrauter Friedrich Wilhelms IV., vorlegte, stimmten die Mitglieder des „Deutschen Vereins" hoffnungsfroh. Der kleindeutsche, unter preuBischer Führung stehende Bundesstaat, so wurde am 26. Mai 1849 zwischen Preußen, Sachsen und Hannover vereinbart, sollte aus einer "Union“ der Fürsten hervorgehen, der sich bald 25 weitere Einzelstaaten anschlossen. Eine Reichsverfassung wurde beraten, die sich an dem Frankfurter Entwurf orientierte, aber ,demokratische“ Elemente weitestgehend eliminierte. Die Fürsten erhielten eine eigene Repräsentation, und an die Stelle des allgemeinen, gleichen und direkten Wahlrechts trat ein Dreiklassenwahlrecht. Die monarchischen Elemente akzeptierte Mommsen gerne, um eine rote Republik zu verhindern. Trotz vollmundiger Bekenntnisse, nicht nur gegen links Front zu machen, wuchs die Kluft zwischen Liberalen und Demokraten. Mit den besten Köpfen der Liberalen hoffte Mommsen, durch die „Union" das hohe Ziel, die Errichtung eines freiheitlichen Nationalstaats, erreichen zu können. Ende Juni 1849 segneten 150 liberale Abgeordnete der Paulskirche in Gotha diese Politik ab und setzten auf dem am 20. März 1850 in Erfurt einberufenen Parlament die Annahme der Unionsverfassung durch. Demokraten und Radikale boykottierten die Wahlen. Doch die liberalen Reden, die auf dem Erfurter Reichstag gehalten wurden, waren nur noch ein Schwanengesang. Faktisch war die Unionspolitik bereits im Herbst des Vorjahres gescheitert, als Österreich wieder erfolgreich seine deutschen Interessen, d. h. den Beitritt der Habsburgermonarchie zum Nationalstaat, vertrat und neben Hannover, Bayern und Württemberg auch Sachsen zum Dreikönigsbund auf Distanz ging.

Mommsen und seine Freunde versuchten in der zweiten Hälfte des Jahres 1849 mit den „Fliegenden Blättern“, die Regierungen von Sachsen und Hannover davon zu überzeugen, daB sie eine besondere Verantwortung für die bundesstaatliche Einigung trügen. Sie verteidigten die kleindeutsche Lösung unter Preußens Führung und polemisierten gegen die „gottlosen Demokraten“ und die „Baalspfaffen“ im allgemeinen und die săchsischen Minister im besonderen. ${ }^{53}$ Die Internationalisienung des Konflikts zwischen Preußen und Öster- 
reich um die Hegemonie in Deutschland lieB diese Artikel jedoch zur Makulatur werden.

Am Ende des Jahres 1850 war die Einheit Deutschlands endgültig in weite Ferne gerückt. Der verachtete Deutsche Bund, gegen den man aufgestanden war, wurde wiederhergestellt. Die liberale Nationalstaatsgründung war gescheitert. Der Staat blieb Obrigkeitsstaat. Mommsen zählte zu den Verlierern. Schon im Juni 1850 klagte er in einem Brief an Henzen bitter, daß „das wunderreiche und wundervolle Jahr 1848 sich in eine einfache Dupierung und eitel Gemeinheit aufgelöst" habe. ${ }^{54}$

Das „wundervolle Jahr 1848 “ hatte auch ein persönliches Nachspiel für Haupt, Jahn und Mommsen. Gegen die aufmüpfigen Professoren, die nicht nur an der Mairevolution 1849 beteiligt gewesen waren, sondern auch später noch die neue, reaktionäre Regienung von Sachsen publizistisch angriffen, wurde ein Strafverfahren eingeleitet. ${ }^{5 s}$ Die Sache zog sich hin. Zunächst sah es so aus, als könne eine Loyalitätserklärung für König und Verfassung genügen. Doch das Kultusministerium wollte das Versprechen, daß man sich der politischen Tätigkeit enthalten werde. Suspension und Amtsenthebung drohten. Mommsen, Jahn und Haupt gaben nicht nach.

Die politische Großwetterlage verschärfte den Konflikt. Die sächsische Regierung, die im Zuge der Abwicklung der 48er Revolution die alten vormärzlichen Stände wieder einberufen hatte, verlangte von der Universität Leipzig die Wahl eines Landtagsdelegierten. Sofort erhob sich Widerspruch, denn durch diese Abgeordnetenwahl hătte die Universität die Wiedereinberufung der alten Stände und die oktroyierte Verfassung anerkannt. Einmal mehr waren es unter den Ordinarien Haupt und Jahn, unter den Extraordinarien Mommsen, die den Protest gegen das Ansinnen der Regierung mittrugen. Schnell war eine überparteiliche Allianz geschlossen. Liberale Anhänger des preußischen Unionsplanes und Vertreter der großdeutschen Lösung fanden zusammen. Die ordentlichen Professoren, die zur Sitzung des akademischen Senats vom 20. Juni 1850 zusammenkamen, wiesen mit 21 gegen 16 Stimmen die Wahlaufforderung zurück. Die Regierung in Dresden antwortete mit Disziplinarverfahren und wollte der Hochschule ein neues Universitätsstatut aufzwingen, das die Machtstellung des Senats und der Ordinarien brechen sollte.

Gegen Haupt, Jahn und Mommsen wurde zudem strafrechtlich vorgegangen. Gegenstand der Verhandlung waren ihre Aktivitäten am 4. Mai 1849. Das Leipziger Appellationsgericht verurteilte Anfang Oktober 1850 in erster Instanz Haupt zu einem Jahr und Mommsen zu neun Monaten Landesgefängnis, weil sie die Bürger Leipzigs aufgefordert hätten, sich zu einer Volksversammlung einzufinden. Dies qualifizierte das Gericht als Vorbereitung zum Hoch- 
verrat. Jahn hingegen wurde aus Mangel an Beweisen freigesprochen. Die Berufungsinstanz hob am 13. Februar des nächsten Jahres auch die gegen Haupt und Mommsen verhängten Strafen auf. Die jungen Männer, hieß es in der Urteilsbegründung, stünden dem praktischen Leben fern und beschäftigten sich bloß mit der Theorie. „Sie leben in Ideen, enthusiasmieren sich für dieselben, ohne die Fähigkeit zu haben, deren praktische Durchführbarkeit beurteilen zu können, und ohne zu bedenken, welches Unheil die versuchte Durchführung derselben mit sich bringen kann. ${ }^{\star 56}$ Mommsen erreichte die Nachricht in Oldesloe: Er war in die alte Heimat zurïckgeeilt, weil sein Vater im Sterben lag.

Der Freispruch bedeutete nicht das Ende der Affäre. Haupt, Jahn und Mommsen wurden aus dem Universitătsdienst entlassen. Beust, der Kultusminister, wies am 22. April 1851 die Universität zur „Entfernung“ der drei Professoren an, weil ihr Verhalten ,in den ersten Tagen des Monats Mai 1849 von der Art gewesen ist, daß sie dadurch öffentliches Ärgernis gegeben, und ein sehr schlechtes Beispiel für die akademische Jugend aufgestellt haben. ${ }^{.57}$ Die disziplinarrechtlich Abgestraften legten Berufung ein. Die Universität wollte die ausgezeichneten Gelehrten halten. Die Buchhändler Härtel, Hirzel, Reimer und Wigand garantierten den Freunden, das entzogene Gehalt zumindest auf ein Jahr aus privaten Mitteln sicherzustellen. ${ }^{58}$ Im August war die Sache entschieden: Das Ministerium wies die Berufung zurück. Die Entlassenen hatten in Leipzig keine Statt mehr. Mommsen mußte sich in den Strom der Flüchtlinge einreihen. Er fand Zuflucht in der Schweiz.

\section{Der politische Professor und der Geschichtsschreiber}

„Eine Revolution ist ein Unglück, aber ein noch größeres Unglück ist eine verunglückte Revolution“, schrieb Heinrich Heine in seinem Pariser Exil, ${ }^{\text {s9 }}$ Durch die „verunglückte" deutsche Revolution des Jahres 1848 wurde eine politische Entwicklung eingeleitet, die die Konvergenz nationaler und liberaler Forderungen auflöste. Einheit durch Recht und Freiheit war das Ziel der Frankfurter Paulskirchen-Versammlung gewesen, das nicht erreicht wurde. 1871 schuf Bismarck schließlich die nationale Einheit von oben „durch Blut und Eisen".

Mommsen mußte wie zahlreiche seiner akademischen Kollegen, mußte wie Gervinus, Droysen, Dahlmann, Waitz, Jahn und Haupt, nach 1848 tiefgehende Orientierungskrisen bewältigen. Die Enttäuschung über die gescheiterte Revolution teilte Mommsen mit vielen seiner Zeitgenossen. Die Erkenntnis, 
daB das politische Wollen nicht genügte, um einen liberalen Staat zu gestalten, schmerzte um so mehr, als die Chance, einen nationalen Verfassungsstaat aufzurichten, zum Greifen nahe gewesen war. Die negative Revolutionserfahrung prägte Mommsens politische Biographie. Das Gefühl der Ohnmacht angesichts der Allmacht der reformunwilligen Eliten saß tief. Threr Macht und ihrer Arroganz setzte er entschlossen das Ideal von Gleichheit und Gerechtigkeit entgegen. Schwierig gestaltete sich sein Verhältnis zu den linken Demokraten und Radikalen, denen er eine Mitverantwortung am Scheitern der Revolution zuwies. Prägend waren zudem die persönlichen Erfahrungen mit der Macht des geschriebenen Wortes, mit dem aktiven Engagement im Verein und mit der Mobilisierung der Bevölkerung. Das intensive Erlebnis der umfassenden Politisierung der Gesellschaft veranlaßte den Bürger Mommsen, an dem Ideal des politischen Professors festzuhalten und zu den spontanen und unmittelbaren, nicht immer rational kalkulierten und strategisch ausgerichteten Politikformen seiner Achtundvierzigerzeit zurückzukehren.

Das animal politicum Mommsen ${ }^{60}$ lebte, wie Friedrich Meinecke einmal treffend für Droysen formulierte, die "Symbiose von Wissenschaft und Politik" ${ }^{61}$ zog sich aber im Gegensatz zu diesem nicht aus der praktischen Politik zurück. Darüber hinaus pochte er auf die ,bildungsbürgerliche‘ Vorherrschaft in der Formulierung und Verwaltung politisch und sozial relevanter Zukunftsbilder. Die liberalen Leitbilder: nationale Identität, rechtliche Gleichheit und politische Partizipation, verteidigte er bis ins hohe Alter.

Unmittelbar nach dem Scheitern der Revolution entdeckte Mommsen wie Droysen und Gervinus ${ }^{62}$ - die Historiographie als politisches Medium. Sie war ihm ein geeignetes Mittel, politische Ansichten einem breiten Publikum zu kommunizieren. Politische Werturteile und historische Kategorien wurden neu aufeinander bezogen. Also beeinflußte die 48er Revolution auch Mommsens Geschichtsschreibung, beeinflußte seine dreibändige „Römische Geschichtec. 63

Mommsen traf mit seinem Werk den Nerv der Zeit. Sein Gegenstand schien deshalb „wahrhaft bedeutsam", weil er ihn ,recht zu schildern" wuBte. ${ }^{64}$ Am römischen Beispiel überprüfte er das Hegelsche Diktum, daß Weltgeschichte „der Fortschritt im BewuBtsein der Freiheit" sei, „ein Fortschritt, den wir in seiner Notwendigkeit zu erkennen haben. ${ }^{\text {"65 }}$ Das Werk war jedoch zugleich mit dem Herzblut des liberalen Achtundvierzigers geschrieben, der das Scheitern der Revolution historiographisch kompensierte und nun einer die Nation einigenden Machtpolitik das Wort redete. Die politischen Auseinandersetzungen seiner Zeit verlegte er in den römischen Senat, damit das gebildete Publikum sich im alten Rom wiederfinden könne. Der Nationalstaat ist für 
Mommsen das Telos der Geschichte, der deutschen wie der römischen. Also breitete er den Mantel des verstehenden Schweigens über Sullas brutale Kriegsführung im Bundesgenossenkrieg, weil er „der wahre und letzte Urheber der vollen staatlichen Einheit Italiens" gewesen sei; und dieser "Gewinn" war „mit endloser Not und Strömen von Blut dennoch nicht zu teuer erkauft."66 Zugleich entwarf er das Idealbild einer römischen Bürgergemeinde, der zwar eine repräsentative Verfassung fehlte, die aber auf bürgerlicher Gleichheit ruhte. Die Utopie der klassenlosen Bürgergesellschaft versetzte er kurzerhand an den Tiber: „Der tiefste und großartigste Gedanke in dem römischen Gemeinwesen war [es], daß es innerhalb der römischen Bürgerschaft keinen Herren und keinen Knecht, keinen Millionär und keinen Bettler geben, vor allem aber der gleiche Glaube und die gleiche Bildung alle Römer umfassen sollte. ${ }^{\alpha 67}$

Mommsens Darstellung vermischte mithin die geschichtliche und die zeitgenössische Perspektive. Die Lebendigkeit und Bildhaftigkeit der aktualisierenden Sprache war jedoch kein Selbstzweck, sondern Mittel der politischen Pädagogik, der letztlich auch die Wissenschaftlichkeit geopfert wurde. Indem Mommsen einen modernen Parteistandpunkt in die römische Geschichte erfolgreich übertrug, bestătigte er Nietzsches Verdikt, daß die Wissenschaft vom Altertum „das Altertum tatsächlich immer nur aus der Gegenwart verstanden“ habe. ${ }^{68}$ Mommsens „Römische Geschichte“ ist weder aktivistische oder voluntaristische Geschichtsschreibung, ${ }^{69}$ sie ist Tendenzhistorie. Eben deshalb wurde sie ein ungeheurer Erfolg.

Mommsen schrieb sein Werk cum ira et studio, und er vergegenwärtigte den historischen Stoff. Die eigene Betroffenheit und Verletztheit machten aus der Geschichte des republikanischen Roms ein Paradigma der historiographie engagée. Ein dialektisches Verhältnis von politischer Parteilichkeit und historischer Objektivităt kennzeichnet die „Römische Geschichte“. Mommsen ist in seiner „Römischen Geschichte“ parteiisch, aber es ist, um Friedrich Gundolf zu zitieren, die Parteilichkeit eines mitstreitenden Agitators und nicht „die eines abseitig nörgelnden Pfaffen."70 


\section{Anmerkungen}

1 Vgl. Wolfgang yon HIPPEL, Revolution im deutschen Sudwesten. Das Großherzogtum Baden 1848/49, Stuttgart/Berlin/Koln 1998, 152ff.

2 Gangolf Húbinger, Georg Gottfried Gervinus, in: Sabine Freitag (Hg.), Die Achtundvierziger. Lebensbilder aus der deutschen Revolution 1848/49, Mänchen 1998, 249-262, Zitat 255.

3 Vgl. etwa Wolfram SiEmann, Die deutsche Revolution von 1848/49, Frankfurt a. M. 1985, 124ff., und WERNER BEST, Soziale Morphologie und politische Orientierungen bildungsbotrgerlicher Abgeordneter in der Frankfurter Nationalversammlung und in der Pariser Assemblée nationale constituante 1848/49, in: Jurgen Kocka (Hg.), Bildungsbilrgertum im 19. Jahrhundert, Teil IV: Politischer Einfluß und gesellschaftliche Formation, Stuttgart 1989, 53-94.

4 Vgl. DieTER LANGEWIESCHE, Bildungsbargertum und Liberalismus im 19. Jahrhundert, in: Kocka (Hg.), Bildungsbargertum (wie Anm. 3), 95-121, Zitat 108.

5 Vgl. SABINE FreItaG, Einleitung, in: Dies. (Hg.), Die Achtundvierziger (wie Anm. 2), 7 .

6 Zum zeithistorischen Hintergrund und zar Geschichte des Liberalismus vgl. Lothar Gall (Hg.), Liberalismus, Koln 1976; DiETER HEIN, Die Revolution von 1848/49, Munchen 1998; DIETER LANGEWIESCHE, Liberalismus in Deutschland, Frankfurt a. M. 1988, 12ff., 39ff.; DeRs (Hg.), Liberalismus im 19. Jahrhundert. Deutschland im europäischen Vergleich, Göttingen 1988; THOMAS NIPPERDEY, Deutsche Geschichte 1800-1866, Munchen 1983, 286ff., 595ff.; WOLFRAM SIEMANN, Die deutsche Revolution von 1848/49, Frankfurt a.M. 1985; HansULRICH WEHLER, Deutsche Gesellschaftsgeschichte, Band 2: Von der Reformära bis zur industriellen und politischen „Deutschen Doppelrevolution". 1815-1845/49, Müchen 1987, 413ff., 703ff.

7 Die nachfolgenden Ausfuhrungen fußen auf meiner Biographie Theodor Mommsens: Stefan Rebench, Theodor Mommsen. Eine Biographie, Munchen 2002, bes. 43ff.; vgl. auch DeRs., Theodor Mommsen und Adolf Harnack. Wissenschaft und Politik im Berlin des ausgehenden 19. Jahrhunderts. Mit einem Anhang: Edition und Kommentierung des Briefwechsels, Berlin/New York 1997, 327ff.

8 Vgl. LOTHAR WICKERT, Theodor Mommsen. Eine Biographie, 4 Bde., Frankfurt a. M. 1959-80, Bd. 1, 175ff.

$9 \mathrm{Zu}$ Mommsens Italienaufenthalt und dem Inschriftenprojekt vgl. WICKERT, Mommsen (wie Anm. 8), I 180ff. und Bd. II, wo in großer Breite Mommsens Reise dokumentiert ist, sowie des weiteren THEODOR MOMMSEN, Tagebuch der französischitalienischen Reise 1844-1845. Mit einem Anhang: Mommsens Denkschrift uber das Corpus Inscriptionum Latinarum 1847, hg. von Gerold und Brigitte Walser, Bern/Frankfurt a.M. 1976 und HeInRICH SCHLANGE-SchONINGEN, Theodor Mommsen in Neapel, in: II Sogno Mediterranea. Tedeschi a Napoli al tempo di Goethe e di Leopardi, Neapel 1996, 136-148 mit weiterer Literatur. Zu den Anfăngen des CIL vgl. auch ADOLF HARNACK, Geschichte der Koniglich Preußischen Akademie der Wissenschaften, 3 Bde. in 4, Berlin 1900, Bd. 1.2, 900ff., und OTTO HIRSCHFELD, Gedächtnisrede auf Theodor Mommsen, in: Abhandlungen der Ko- 
niglich Preußischen Akademie der Wissenschaften, Berlin 1904, 1025-1060, zitiert nach: Ders., Kleine Schriften, Berlin 1913, 931-965, hier 932ff.

10 Zitiert nach WICKERT, Mommsen (wie Anm. 8), II 198. Wickerts Zweifel an der Authentizităt der von Christian Hulsen uberlieferten Aussage teile ich nicht.

$11 \mathrm{Zu}$ Mommsens Ruckkehr aus Italien und seiner journalistischen Tătigkeit vgl. WICKERT, Mommsen (wie Anm 8), II 200ff. und III lff., sowie LUDO MORITZ Hartmann, Theodor Mommsen. Ein biographische Skizze. Mit einem Anhange: Ausgewăhlte politische Aufsătze Mommsens, Gotha 1908, 33ff., und ALFRED HEuss, Theodor Mommsen und das 19. Jahrhundert, Kiel 1956, $137 \mathrm{ff}$.

12 Zum Hintergrund vgl. JOHANNES JENSEN, Nordfriesland in den geistigen und politischen Stromungen des 19. Jahrhunderts: 1797-1864, Neumunster 1961 (ND Bräist/Bredstedt 1993); Olaf Klose und Christian Degn, Die Herzogtumer im Gesamtstaat 1720-1830, Neumunster 1960; NIPPERDEY, Deutsche Geschichte 1800-1866 (wie Anm. 6), 622ff;; ALEXANDER SCHARFF, Schleswig-Holstein und die Auflosung des dănischen Gesamtstaates 1830-1864/1867, Neumünster 1975; SIEMANN, Revolution (wie Anm. 6), 153ff;; GONTER WollsteIN, Das Großdeutschland der Paulskirche. Nationale Ziele in der bürgerlichen Revolution 1848/49, Dusseldorf 1977, 23ff., sowie STEEN Bo FrandSEN, Dănemark - Der kleine Nachbar im Norden. Aspekte der deutsch-dänischen Beziehungen im 19. und 20. Jahrhundert, Darmstadt 1994, 48ff., und VAGN SKOVGAaRD-PETERSEN, Danmarks historie, Bd. 5: Tiden 1814-1864, Kopenhagen 1985.

$13 \mathrm{Zu}$ Theodor. Storm vgl. Georg Bollenbeck, Theodor Storm. Eine Biographie, Frankfurt a. M. 1988; KarL ERNST LAage, Theodor Storm. Leben und Werk, Husum ${ }^{6} 1993$; HARTMUT VInçON, Theodor Storm in Selbstzeugnissen und Bilddokumenten, Reinbek ${ }^{13}$ 1994; REGINA FASOLD, Theodor Storm, Stuttgart/Weimar 1997; DAVID A. JACKSON, Theodor Storm. Dichter und demokratischer Humanist. Eine Biographie, Berlin 2001 mit weiterer Literatur.

14 Vgl. WICKERT, Mommsen (wie Anm. 8), III 7.

15 Wichtige Artikel, die Mommsen in der „Schleswig-Holsteinischen Zeitung“ 1848 schrieb, sind nachgedruckt bei HARTMANN, Mommsen (wie Anm. 11), 161-254; vgl. zudem die Obersicht bei CARL GEHRCKE, Theodor Mommsen als schleswigholsteinischer Publizist (mit einem Anhang politischer Mommsen-Aufsatze), Breslau 1927, 62-64, und den Abdruck weiterer Artikel im Anhang ebd. 154-203. Zu Einwănden gegen die Zuweisung anonym verfaßter Artikel an Mommsen vgl. WiCKERT, Mommsen (wie Anm. 8), III 423.

16 THEODOR MOMMSEN, Die Schlacht bei Schleswig, in: Schleswig-Holsteinische Zeitung 1848, Nr. 9 vom 25. April, 32-34; wiederabgedruckt in: Ders., Reden und Aufsatze, hg. von Otto Hirschfeld, Berlin 1905, 363-372. Vgl. auch Theodor Mommsen als Schriftsteller. Ein Verzeichnis seiner Schriften von KARL ZANGEMEISTER. Im Auftrage der Koniglichen Bibliothek bearbeitet und fortgesetzt von EMIL JACOB. Neu bearbeitet von STEFAN REBENICH, Hildesheim 2000, Nr. 119.

17 THEODOR MOMMSEN, Unsere Wahlen zum Nationalparlament, in: SchleswigHolsteinische Zeitung 1848, Nr. 8 vom 24. April, wiederabgedruckt in: HARTMANN, Mommsen (wie Anm. 11), 162-171. 
18 THEODOR MOMMSEN, Versammlung der Centralwahlcommittee in Neumunster, in: Schleswig-Holsteinische Zeitung 1848, Nr. 11 vom 27. April; vgl. HARTMANN, Mommsen (wie Anm. 11), 35.

19 Vgl. hierzu auch Ulrike von HirschHAusEn, Liberalismus und Nation. Die Deutsche Zeitung 1847-1850, Dusseldorf 1998, die exemplarisch die Programmatik, die organisatorischen Strukturen sowie die soziale Rekrutierung der Mitarbeiter und der Leser am fuhrenden Blatt der deutschen Konstitutionellen wahrend der Revolutionsjghre untersucht.

20 Vgl. ALFRED Heuss, Niebuhr und Mommsen. Zur wissenschaftsgeschichtlichen Stellung Theodor Mommsens, in: A\&A 14, 1968, 1-18 ( $=$ Ders., Gesammelte Schriften 3, Stuttgart 1995, 1699-1716), und GERRrT WALTHER, Niebuhrs Forschung, Stuttgart 1993.

21 Zitiert nach HaRTMANn, Mommsen (wie Anm. 11), 36; vgl. auch GeHRCKE, Mommsen (wie Anm. 15), 74.

22 Vgl. UlRICH MuHLACK, Johann Gustav Droysen, in: Freitag (Hg.), Die Achtundvierziger (wie Anm. 2), 263-276. Zum Hintergrund vgl. Mommsens Briefwechsel mit Droysen und Wilhelm Ahlmann, dem Korrespondenten der Schleswig-Holsteinischen Zeitung in Frankfurt: JOHANN Gustav DroYsEn, Briefwechsel, 2 Bde., hg. von Rudolf Habner, Berlin/Leipzig 1929 (= Deutsche Geschichtsquellen des 19. Jahrhunderts, Bd. 25/26).

23 TheOdor MOMmSEN, Die Annexion Schleswig-Holsteins. Ein Sendschreiben an die Wahlmånner der Stadt Halle und des Saalkreises, Berlin 1865, zitiert nach MOMmSEN, Reden und Aufsătze (wie Anm. 16), 373-401.

$24 \mathrm{Vgl}$. HÜBnger, Gervinus (wie Anm. 2), 257.

25 Zur historischen Einordnung der joumalistischen Beitrăge von Mommsen vgl. auch GEHRCKE, Mommsen (wie Anm. 15), 65ff., und Heuss, Mommsen (wie Anm. 11), 140ff.

26 THEODOR MOMMSEN, Unsere Wahlen zum Nationalparlament, zitiert nach HARTMANN (wie Anm. 11), 164.

27 Vgl. REBENICH, Theodor Mommsen. Eine Biographie (wie Anm. 7), 107ff.

28 Vgl. HúBnaER, Gervinus (wie Anm. 2), 258.

29 THEODOR MOMMSEN, Die Verbesserung der Lage der arbeitenden Klassen, in: Schleswig-Holsteinische Zeitung, Nr. 32 vom 22. Mai 1848, zitiert nach GEHRCKE, Mommsen (wie Anm. 15), 170-174.

30 Vgl. GeHRCKE, Mommsen (wie Anm. 15), 89f.

31 Vgl. GeHRCKE, Mommsen (wie Anm. 15), $91 \mathrm{ff}$,, sowie die von HarTManN, Mommsen (wie Anm. 11), 183ff. abgedruckten Artikel.

32 Vgl. GeHrCKe, Mommsen (wie Anm. 15), 99, und WickERT, Mommsen (wie Anm. 8), III 21ff.

33 Vgl. Gehrcke, Mommsen (wie Anm. 15), 101ff., 185ff., sowie Hartmann, Mommsen (wie Anm. 11), 216ff.

34 Vgl. seinen Brief an Wilhelm Henzen vom 5. November 1848 bei WICKERT, Mommsen (wie Anm. 8), III 28.

35 So Mommsen in einem Brief an Otto Jahn vom 5. August 1848, zitiert nach LOTHAR WicKeRT (Hg.), Theodor Mommsen - Otto Jahn. Briefwechsel 18421868, Frankfurt a. M. 1962, 72. 
36 Vgl. WicKERT, Mommsen (wie Anm. 8), III 23ff.

$37 \mathrm{Zu}$ Mommsens politischer und wissenschaftlicher Tátigkeit in Leipzig vgl. HaRTMANN, Mommsen (wie Anm. 11), 43ff.; Heuss, Mommsen (wie Anm. 11), 157ff., 264ff., und WICKERT, Mommsen (wie Anm. 8), III 152ff., 479ff. Zum zeithistorischen Hintergrund und zur Geschichte der Leipziger Universität vgl. die in Anm. 6 genannte Literatur sowie KoNRAD KRAUSE, Alma mater Lipsiensis. Geschichte der Universităt Leipzig von 1409 bis zur Gegenwart, Leipzig 2003, $111 \mathrm{ff}$; Deter LangewIesche, Die Anfänge der deutschen Parteien. Partei, Fraktion und Verein in der Revolution 1848/49, in: Geschichte und Gesellschaft 4, 1978, 324361; WINFRIED LOSCHBURG, Der Widerstand der Universităt Leipzig gegen die Reaktivierung der alten Stände in Sachsen im Jahre 1850, in: Karl-Marx-Universität Leipzig 1409-1959, 2 Bde., Leipzig 1959, Bd. 1, 312-327; HEDE THIELBEER, Universităt und Politik in der Deutschen Revolution von 1848, Bonn 1983, 25ff.; RoLF WEBER, Die Universităt Leipzig in der Revolution 1848/49, in: Karl-MarXUniversităt Leipzig 1409-1959, 2 Bde., Leipzig 1959, Bd. 1, 250-311, und Ders., Die Revolution in Sachsen 1848/49, Entwicklung und Analyse ihrer Triebkrafte, Berlin 1970.

38 Jahn an Mommsen am 7. August 1848, zitiert nach WICKERT (Hg.), MommsenJahn (wie Anm. 35), 74.

39 Zur Weidmann'schen Buchhandlung und der Familie Reimer vgl. ERNST VOLLERT, Die Weidmannsche Verlagsbuchhandlung in Berlin 1680-1930. Mit einem Geleitwort von W. Georg Olms und einer Ergänzung: Die Weidmannsche Verlagsbuchhandlung 1930-1983 von W. Joachim Freyburg, Hildesheim 1983.

40 Zum Leipziger Freundeskreis vgl. WICKERT, Mommsen (wie Anm. 8), III 35ff., sowie OTro JAHN, Biographische Aufsătze, Leipzig 1866, 165-220; RICHARD SCHONE, Erinneringen an Theodor Mommsen zum 30. November 1917. Herausgegeben von Hermann Schðne, Münster 1923, $11 \mathrm{ff}$.

41 Vgl. WeBER, Universität (wie Anm. 37), 266ff.

42 Zitiert nach WEBER, Universităt (wie Anm. 37), 268.

43 Vgl. WEBER, Universitat (wie Anm. 37), 287.

44 THEODOR MOMMSEN, Die Grundrechte des deutschen Volke. Mit Belehrungen und Erlăuterungen, Leipzig 1849 (= Die Grundrechte des deutschen Volkes Belehrungen und Erläuterungen [1849], hg. von Lothar Wickert, Frankfurt a. M. 1969); vgl. Theodor Mommsen als Schriftsteller (wie Anm. 16), Nr. 128a.

45 Adresse an den Staatsminister Dr. von der Pfordten. Leipzig, den 23. Januar 1849, in: Leipziger Tageblatt 1849, 24. Januar, 279; vgl. Theodor Mommsen als Schriftsteller (wie Anm. 16), Nr. 120; zitiert nach WickeRT, Mommsen (wie Anm. 8), III 154.

46 Zitiert nach WICKERT, Mommsen (wie Anm. 8), III 481.

47 Zitiert nach WICKERT, Mommsen (wie Anm. 8), II 154.

48 Vgl. WeBER, Universităt (wie Anm. 37), 296ff.

49 Vgl. WICKERT, Mommsen (wie Anm. 8), III 156.

50 Zitiert nach WEBER, Universitat (wie Anm. 37), 299.

51 Vgl. hierzu sowie zum folgenden die in Anm. 37 genannte Literatur.

S2 Zitiert nach WICKERT, Mommsen (wie Anm. 8), III 162. 
53 Vgl. Theodor Mommsen als Schriftsteller (wie Anm. 16), 121, sowie Heuss, Mommsen (wie Anm. 11), 157ff., 264ff., und WiCKERT, Mommsen (wie Anm. 8), III 160ff. (Zitate 165).

54 Zitiert nach WICKERT, Mommsen (wie Anm. 8), III $167 f$.

55 Vgl. zum folgenden WICKERT, Mommsen (wie Anm. 8), III 173ff., sowie Mommsens Personalakte im Universitătsarchiv Leipzig (Rep. I/VIII/204), die Dr. Gerald Wiemers mir freundlicherweise zugänglich gemacht hat.

56 Vgl. WiCKERT, Mommsen (wie Anm. 8), III 184; vgl. WEBER, Universităt (wie Anm. 37), 308f.

57 Vgl. WICKERT, Mommsen (wie Anm. 8), III 185.

58 Vgl. WiCKERT, Mommsen (wie Anm. 8), III 186.

59 HEINRICH HEINE, Ludwig Börne. Eine Denkschrift, in: Ders., Historisch-kritische Gesamtausgabe der Werke, Bd. 11, hg. von Helmut Koopmann, Hamburg 1978, 75.

$60 \mathrm{Zu}$ Mommsens Selbstbezeichnung als animal politicum vgl. REBENICH, Mommsen und Harnack (wie Anm. 7), 327ff. mit weiterer Literatur.

61 FRIEDRICH MEINECKE, Johann Gustav Droysen. Sein Briefwechsel und seine Geschichtsschreibung, in: Ders., Zur Geschichte der Geschichtsschreibung, hg. von Eberhard Kessel, München 1968, 125-167, Zitat 125.

62 Vgl. Gangolf HüBINGER, Georg Gottfried Gervinus. Historisches Urteil und politische Kritik, Göttingen 1984.

63 Zur ersten Orientierung sei verwiesen auf die vorzügliche Einfuthrung von KARL Chirust, Theodor Mommsen und die „Romische Geschichte ", im achten Band der dtv-Ausgabe der „Rסmischen Geschichte“, München 1976 ('2001), 7-66 (= KARL. CHRIST, Romische Geschichte und Wissenschaftsgeschichte 3, Darmstadt 1983, 26-73). Aus der umfangreichen gelehrten Literatur seien genannt: KLAus BRINGMANn, Das Problem einer „Römischen Revolution“, in: Geschichte in Wissenschaft und Unterricht 31, 1980, 354-377; KARL CHRIST, Caesar. Annăherungen an einen Diktator, Munchen 1994, 134ff.; JOACHIM FEST, Wege zur Geschichte. Uber Theodor Mommsen, Jacob Burckhardt und Golo Mann, Zarich 1992, 29ff.; ALFRED Heuss, Theodor Mommsen als Geschichtsschreiber, in: Notker Hammerstein (Hg.), Deutsche Geschichtswissenschaft um 1900, Stuttgart 1988, 37-95 (= Ders., Gesammelte Schriften 3, Stuttgart 1995, 1744-1802); Heuss, Mommsen (wie Anm. 11), 58ff; HANS KLOFT, Caesar und die Legitimität. Uberlegungen zum historischen Urteil, in: Archiv fur Kulturgeschichte 64/65, 1982/83, 1-39; JAMES F. MCGLEw, Revolution and Freedom in Theodor Mommsen's „Römische Geschichte", in: Phoenix 40, 1986, 424-445; Christian MEIER, Das Begreifen des Notwendigen. Zu Theodor Mommsens Romischer Geschichte, in: Reinhart Koselleck u. a. (Hgg.), Formen der Geschichtsschreibung, München 1982, 201-244, HENRIK MourrTsen, Italian Unification. A Study in Ancient and Modern Historiography, London 1998, 23ff;; WILFRED NIPPEL, Theodor Mommsen, in: Volker Reinhardt (Hg.), Hauptwerke der Geschichtsschreibung, Stuttgart 1997, 436-440; ELISABETH TORNOW, Der Revolutionsbegriff und die spăte romische Republik. Eine Studie zur deutschen Geschichtsschreibung im 19. und 20. Jh., Frankfurt a. M. 1978; GERRIT WALTHER, Theodor Mommsen und die Erforschung der romischen Geschichte, in: Aporemata 5, 2001, 241-258; WICKERT, Mommsen (wie Anm. 8), III 399ff., 
618ff.; ALBERT WUCHER, Theodor Mommsen. Geschichtsschreibung und Politik, Göttingen ${ }^{2} 1968$, sowie STEFAN RfBENTCH, Theodor Mommsens „Römische Geschichte“", erscheint in: Elke Stein-Hölkeskamp/Karl-Joachim Hölkeskamp (Hgg.), Erinnerungsorte der römischen Antike. Rom und sein Imperium, München 2005.

64 Vgl. HANS-GEORG GADAMER, Wahrheit und Methode, Tubingen ${ }^{4} 1975,268$.

65 Vgl. GOTTFRIED WILHELM FRIEDRICH HEGEL, Vorlesungen uber die Rechtsphilosophie, Bd. 3, Stuttgart 1974, 821; DERS., Vorlesungen über die Philosophie der Geschichte (= Werke in zwanzig Bänden; Bd. 12), Frankfurt a. M. 1970, 29ff., $378 f f$.

66 THEODOR MOMMSEN, Romische Geschichte, Bd. 2, Berlin ${ }^{9} 1903,373$.

67 THEODOR MOMMSEN, Romische Geschichte, Bd. 1, Berlin ${ }^{9} 1902,884$.

68 FrIEDRICH NIETZSCHE, Wir Philologen, in: Ders., Werke in fünf Bănden, Bd. 5, München/Wien 1980, 325.

69 Vgl. etwa Wucher, Mommsen (wie Anm. 63), 25, 215, und HEuss, Mommsen (wie Anm. 11), 68.

70 FRIEDRICH GuNDOLF, Caesar im neunzehnten Jahrhundert, Berlin 1926, 58. 